\title{
Espondilodiscitis: experiencia clínica en un hospital general de Chile
}

\author{
Andrés Soto, Alberto Fica, Jeannette Dabanch, Felipe Olivares y Lorena Porte
}

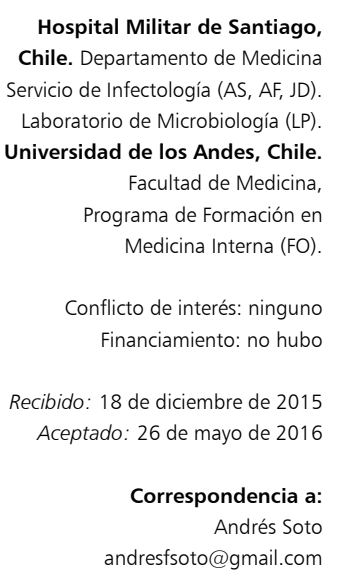

.

\section{Spondylodiscitis: clinical experience in a Chilean general hospital}

Background: Spondylodiscitis (SD) involves long periods of hospitalization, diagnostic latency and risk of longterm complications. No updated series are available in Chile and a change in demographic features and etiology is suspected. Aim: To characterize a group of patients with SD. Patients and Methods: Clinical series including patients over an 8 year period. Results: We identified 37 patients; $37.8 \%$ women and $62.2 \%$ men (mean age 66.8 years); $64.9 \%$ were elderly; $35.1 \%$ had diabetes and $21.6 \%$ urological comorbidity. Main symptoms were pain and fever. Erythrocyte sedimentation rate was elevated in $89.2 \%$, and $86.5 \%$ patients had MRI, which was always confirmatory. Lumbar spine was the most common site of infection (43.2\%). Etiology was identified in 28/37 patients: $71.4 \%$ yielded grampositive cocci (Staphylococcus aureus predominantly), Mycobacterium tuberculosis was identified in only $10.7 \%$. Staphylococcus aureus was associated to medical comorbidities $(\mathrm{p}<0,05)$ and gramnegative bacilli to hepatobiliar or intestinal symptoms $(p<0,05)$. Culture obtained by a surgical procedure had the highest yield. The average duration of antibiotic therapy was 63.8 days (IQR 53-72). Treatment-related side effects were detected in $18.9 \%$ of patients. The average hospital stay was 38.9 days. No deaths occurred during hospitalization. Motor sequelae were present in $18.9 \%$ of this series. Discussion: Most patients with SD were older adults. Staphylococcus aureus was predominant and M. tuberculosis was uncommon. Antibiotic side effects were relevant as well as the neurological complications.

Key words: Spondylodiscitis, vertebral osteomyelitis, spondylitis, bacteremia, Staphylococcus aureus.

Palabras clave: Espondilodiscitis, osteomielitis vertebral, espondilitis, bacteriemia, Staphylococcus aureus.

\section{Introducción}

$\mathrm{E}$ spondilodiscitis (ED) es la afección espinal de origen infeccioso que puede involucrar a cuerpos vertebrales, espacios y discos intervertebrales adyacentes, como parte del mismo proceso patológico ${ }^{1,2}$. Generalmente, en el concepto de ED se incluyen los términos de osteomielitis vertebral, discitis y espondilitis ${ }^{1-3}$.

Esta infección es causada principalmente por agentes bacterianos piógenos, entre los más comunes Staphylococcus aureus, Streptococcus spp. y algunas enterobacterias, micobacterias y, en algunos raros casos, por hongos o parásitos, los que ingresan al tejido óseo y el disco intervertebral, preferentemente por vía hematógena ${ }^{1-4}$.

La ED generalmente está asociada a condiciones de riesgo como edad avanzada, diabetes mellitus, cirrosis hepática, la existencia de un dispositivo intravascular y la presencia de endocarditis infecciosa (EI) concomitante, entre otras ${ }^{1,5,6}$. Si bien se presenta en forma infrecuente en la población (se estima en sólo 3-5\% del total de las osteomielitis) $)^{1}$, implica prolongados tiempos de latencia diagnóstica y períodos de estadía hospitalaria y frecuencias variables de obtención de un agente etiológico, lo que aumenta la morbilidad asociada y el riesgo de complica- ciones y/o secuelas a corto y largo plazo, principalmente de origen neurológico $0^{2,3,6}$.

Al día de hoy disponemos de pocas publicaciones chilenas respecto a series de pacientes con ED y la mayoría data de más de una décadaa ${ }^{7,8}$. En la última experiencia reportada, la principal etiología fue Mycobacterium tuberculosis (entidad conocida como Mal de Pott) ${ }^{7}$, aunque hay algún reporte reciente enfocado en aspectos pronósticos?. Dado los cambios demográficos que ha experimentado progresivamente nuestra población, se hace necesario conocer las características actuales de los pacientes que cursan con ED.

En consecuencia, se efectuó un estudio de ED en pacientes adultos en un hospital general de Chile, con la idea de conocer sus actuales características clínicas, epidemiológicas, etiologías, tratamiento y pronóstico, así como determinar factores de riesgo para ED por determinados agentes infecciosos y la presencia secuelas neurológicas.

\section{Pacientes y Métodos}

\section{Diseño del estudio y pacientes}

Estudio descriptivo, retrospectivo, tipo serie clínica. Se revisó las fichas de los pacientes con diagnóstico de ED, 
realizado entre enero de 2006 y diciembre de 2013 en el Hospital Militar de Santiago, Chile (HMS), con pesquisa de éstos a través de dos estrategias: la búsqueda activa de egresos hospitalarios en el Departamento de Bioestadística de nuestro hospital, utilizando los códigos respectivos según la Clasificación Estadística Internacional de Enfermedades (CIE-10) para los diagnósticos osteomielitis vertebral (M46.2), infección del disco intervertebral (M46.3), discitis (M46.4), espondilopatías infecciosas (M46.5), tuberculosis vertebral (M49.0), espondilitis por brucelosis (M49.1) y espondilitis por enterobacterias (M49.2); y a través del registro interno del Servicio de Infectología de pacientes hospitalizados y evaluados con dicho diagnóstico. El estudio fue aprobado por el Comité de Ética del HMS.

\section{Criterios de inclusión}

Se incluyeron pacientes con diagnóstico de ED en base a criterios clínicos y radiológicos, con o sin confirmación microbiológica y realizado, ya fuese por un médico internista, traumatólogo, neurocirujano y/o infectólogo, entre enero de 2006 y diciembre de 2013.

\section{Caracterización clínica y análisis de datos}

Los casos identificados fueron analizados mediante una pauta estructurada que incluyó datos demográficos, condiciones predisponentes y co-morbilidades asociadas a ED, manifestaciones clínicas, resultados de estudio de laboratorio e imágenes, de estudios microbiológicos e histológicos y aspectos terapéuticos. Se incluyó además el lugar de adquisición de la ED: comunitaria o nosocomial. Se definió esta última como aquel evento que aparece luego de algún procedimiento diagnóstico o terapéutico en el mismo sitio de infección, en los últimos 12 meses, o que aparece durante o después de la misma hospitalización. Se determinó también la presencia de complicaciones durante la hospitalización y/o a largo plazo. Se ingresaron los datos recopilados en una planilla de registro y al software SPSS para su análisis.

\section{Análisis estadístico}

La información de variables categóricas y continuas fue resumida en forma descriptiva y tabulada o presentada en gráficos. Se buscaron factores asociados a infecciones por S. aureus, Enterococcus spp. y bacilos gramnegativos, mediante tablas de contingencia con variables categóricas o continuas categorizadas y aplicando pruebas no paramétricas (prueba bilateral de Fischer). Se hizo un análisis similar para la búsqueda de factores asociados a secuelas neurológicas.

\section{Resultados}

\section{Características demográficas y epidemiológicas}

Entre los años 2006 y 2013, se identificaron 37 pacientes con diagnóstico de ED, de los cuales 23 (62,2\%) co- rrespondieron a hombres y 14 (37,8\%) a mujeres, con un promedio etario de 66,8 años (rango 19-86). El promedio de estadía hospitalaria fue 38,9 días. Un $86,5 \%$ se consideró de adquisición comunitaria y la fracción restante $(13,5 \%)$ infección asociada a la atención en salud (IAAS) (Tabla 1). Entre las condiciones y co-morbilidades asociadas se identificaron una edad avanzada (edad $\geq 65$ años, $(64,9 \%)$, diabetes mellitus tipo 1 o $2(35,1 \%)$, historia previa de procedimientos urológicos invasores (catéteres tipo urinario permanente o doble $\mathrm{J}$, o nefrostomías) (21,6\%), cirrosis hepática $(16,2 \%)$ y cirugía de columna reciente $(13,5 \%)$. Otras condiciones predisponentes incluyeron la presencia de una dispositivo intravascular, antecedentes de tuberculosis (TBC) pulmonar antigua, endocarditis infecciosa (EI), y diálisis crónica. Sólo en 5,4\% no había factores predisponentes conocidos (Tabla 2).

Tabla 1. Características demográficas y clínicas de 37 pacientes con espondilodiscitis, atendidos en el Hospital Militar de Santiago entre los años 2006 y 2013

\begin{tabular}{|c|c|}
\hline Variable & Resultado \\
\hline Edad promedio (rango etario) & $66,8(19-86)$ \\
\hline$>65$ años, $n^{\circ}(\%)$ & $24 \quad(64,9)$ \\
\hline \multicolumn{2}{|l|}{ Sexo, $n^{\circ}(\%)$} \\
\hline Masculino & $(62,2)$ \\
\hline Femenino & $(37,8)$ \\
\hline \multicolumn{2}{|l|}{ Lugar de adquisición, n (\%) } \\
\hline Comunitaria & $(86,5)$ \\
\hline Asociada a la atención en salud & $(13,5)$ \\
\hline Estadía hospitalaria promedio, días (IQR)* & $38,9(23-57)$ \\
\hline
\end{tabular}

Tabla 2. Condiciones predisponentes y co-morbilidades asociadas en 37 pacientes con espondilodiscitis, atendidos en el Hospital Militar de Santiago entre los años 2006 y 2013

\begin{tabular}{lcc}
\hline Condiciones $\mathbf{y}$ co-morbilidades asociadas & $\mathbf{n / N}$ & $\%$ \\
\hline Diabetes mellitus tipo 1 o 2 & $13 / 37$ & 35,1 \\
\hline Procedimientos urológicos invasores & $8 / 37$ & 21,6 \\
\hline Cirrosis hepática & $6 / 37$ & 16,2 \\
\hline Cirugía de columna reciente & $5 / 37$ & 13,5 \\
\hline Dispositivo intravascular* & $3 / 37$ & 8,1 \\
\hline Tuberculosis pulmonar antigua & $2 / 37$ & 5,4 \\
\hline Endocarditis infecciosa concomitante & $2 / 37$ & 5,4 \\
\hline Enfermedad renal en diálisis crónica** & $1 / 37$ & 2,7 \\
\hline Sin condición asociada & $2 / 37$ & 5,4 \\
\hline
\end{tabular}

*Definido como catéter venoso central, dispositivo intracardíaco o presencia de endoprótesis vascular. **Diálisis por fístula arterio-venosa. 


\section{Manifestaciones clínicas}

El síntoma más frecuente fue el dolor axial $(83,8 \%)$, seguido de compromiso neurológico $(37,8 \%)$, caracterizado por paraparesia, paraplejia o retención urinaria. Hubo baja de peso significativa en $29,7 \%$ y fiebre en $21,6 \%$. La duración promedio de los síntomas hasta el diagnóstico de ED fue de 60,6 días (Tabla 3).

\section{Hallazgos de laboratorio y radiológicos}

Un 89,2\% presentó elevación de la velocidad de eritrosedimentación (VHS) (promedio: $64 \mathrm{~mm} / \mathrm{h}$ ) y 77,1\% de la proteína $\mathrm{C}$ reactiva (promedio: $59 \mathrm{mg} / \mathrm{L}$ ). Hubo leucocitosis o leucopenia en 37,8\% y anemia en 59,5\% de los pacientes, siempre normocítica normocrómica. Otros hallazgos incluyeron elevación de fosfatasas alcalinas e

Tabla 3. Signos y síntomas presentes en $\mathbf{3 7}$ pacientes con espondilodiscitis, atendidos en el Hospital Militar de Santiago entre 2006 y 2013

\begin{tabular}{lr}
\hline Manifestaciones clínicas & Resultado \\
\hline $\begin{array}{l}\text { Motivo de consulta, } \mathrm{n}^{\circ}(\%) \\
\text { Dolor }\end{array}$ & $31(83,8)$ \\
Fiebre & $8(21,6)$ \\
\hline $\begin{array}{l}\text { Síntomas asociados } \\
\text { Baja de peso } \\
\text { Compromiso neurológico }\end{array}$ & $11(29,7)$ \\
\hline Duración de síntomas, días (IQR) ${ }^{*}$ & $14(37,8)$ \\
\hline *IQR: interval intercuartil. & $60,6(12-120)$ \\
\hline
\end{tabular}

Tabla 4. Agentes etiológicos confirmados, en 28 pacientes con espondilodiscitis, atendidos en el Hospital Militar de Santiago entre 2006 y 2013

\begin{tabular}{|c|c|c|c|}
\hline Agente infeccioso & $n / N$ & $\%$ & Comentarios \\
\hline \multicolumn{4}{|l|}{ Cocáceas grampositivas } \\
\hline Staphylococcus aureus & $9 / 28$ & 32,1 & Una cepa con resistencia a oxacilina \\
\hline Enterococcus faecalis & $6 / 28$ & 21,4 & Sin endocarditis infecciosa concomitante \\
\hline Staphylococcus coagulasa negativa & $3 / 28$ & 10,7 & Dos cepas con resistencia a oxacilina \\
\hline Streptococcus agalactiae & $1 / 28$ & 3,6 & \\
\hline Streptococcus grupo viridans & $1 / 28$ & 3,6 & Sin endocarditis infecciosa concomitante \\
\hline \multicolumn{4}{|l|}{ Bacilos gramnegativos } \\
\hline Escherichia coli & $3 / 28$ & 10,7 & \\
\hline Pseudomonas aeruginosa & $1 / 28$ & 3,6 & Cepa multisensible. Caso comunitario \\
\hline Serratia marcescens & $1 / 28$ & 3,6 & Paciente con múltiples co-morbilidades \\
\hline Mycobacterium tuberculosis & $3 / 28$ & 10,7 & Ningún caso con TBC en otro sitio \\
\hline
\end{tabular}

hipoalbuminemia, en 20,6 y $34,4 \%$, respectivamente. En 23 de los 37 casos $(62,2 \%)$ se contó con tomografía axial computada (TAC) de columna, que reveló hallazgos compatibles con ED en 78,3\%. Sólo en 16 casos (43,2\%) se realizó cintigrafía ósea, la que mostró a su vez hallazgos sugerentes (ej: aumento de la actividad osteoblástica e hipercaptación focal) en 93,8\%. En 32 pacientes $(86,5 \%)$ se realizó resonancia magnética $(\mathrm{RM})$ de columna, la que localizó y confirmó el diagnóstico en todos los pacientes (sensibilidad 100\%). A través de TAC y/o RM (incluyendo con esto al 100\% del grupo) se pesquisaron complicaciones locales, como colecciones o abscesos intra-raquídeos o paravertebrales en $29,7 \%$ y compromiso radicular en $48,6 \%$. La presentación más frecuente fue unifocal (dos vértebras contiguas y un disco comprometidos) en $73 \%$, siendo más frecuente en la columna lumbar y lumbo-sacra $(43,2 \%)$, seguida de torácica $(27,0 \%)$ y sólo en un caso cervical. Hubo compromiso multifocal $(\geq 2$ espacios intervertebrales comprometidos) en $24,3 \%$. En $75,7 \%$ de los pacientes se realizó ecocardiografía doppler, objetivándose en forma concomitante a la ED dos casos con hallazgos ecográficos sugerentes de EI.

\section{Estudio microbiológico e histológico}

Se identificó agente etiológico en 28 de los 37 pacientes $(75,7 \%)$. De éstos, $71,4 \%$ aislados por hemocultivos en siete casos, por cultivo de punción vertebral en 11 casos y por ambos métodos en siete (Tabla 4). Staphylococcus aureus fue la principal etiología (32,1\%), presentando resistencia a oxacilina en uno de los nueve aislados. El segundo agente causal fue Enterococcus faecalis en 21,4\%. Escherichia coli se presentó con una frecuencia de $10,7 \%$. (Tabla 4). El método diagnóstico de mayor rendimiento fue el cultivo de tejido obtenido por punción vertebral quirúrgica abierta $(73,1 \%)$, seguido por hemocultivos aerobios obtenidos de sangre periférica (53,8\%). Los cultivos obtenidos por punción vertebral dirigida por visión tomográfica sólo obtuvieron una positividad de $33,3 \%$ en esta serie (Figura 1). Por otra parte, se demostró en tres de los 28 pacientes con diagnóstico etiológico una ED tuberculosa, diagnosticada por cultivo de Koch (un caso) o histología (dos casos), todos con baciloscopias de tejido negativas. Además, hubo tres pacientes con etiología no precisada por métodos convencionales en los que se efectuó estudio molecular complementario: reacción de polimerasa en cadena (RPC) convencional para M. tuberculosis en un caso y RPC bacteriana universal 16S ARNr en dos casos, todos con resultado negativo. No se objetivó casos de etiología polimicrobiana.

Finalmente, el estudio histológico de tejido óseo fue realizado en 25 de 37 pacientes $(67,6 \%)$, y en sólo 18 casos el resultado pudo ser obtenido para este análisis: 14 (77,8\%) fueron compatibles con ED piógena, dos 
$(11,1 \%)$ con ED tuberculosa, y dos $(11,1 \%)$ sin hallazgos histológicos de ED (en estos últimos casos el diagnóstico fue microbiológico).

\section{Factores de riesgo para $E D$, según agentes etiológicos}

En el análisis univariado, se encontró una asociación significativa entre co-morbilidad de tipo médica (incluyendo diabetes mellitus, cirrosis hepática y endocarditis concomitante) y ED por $S$. aureus (prueba bilateral de Fisher p: 0,011). No se encontró asociación entre edad, co-morbilidades quirúrgicas, tabaco, $\mathrm{OH}$, cirugía, enfermedad renal en diálisis crónica, presencia de fiebre o diaforesis, duración de síntomas o adquisición nosocomial. Hubo una asociación significativa entre cirrosis hepática e infecciones por bacilos gramnegativos (enterobacterias y no fermentadores) $(\mathrm{p}<0,05)$, y también entre procedimientos urológicos y este grupo bacteriano $(\mathrm{p}<0,05)$. Además, se apreció una tendencia a que estos pacientes tuvieran síntomas y evolución de menor tiempo $(<30$ días; p: 0,057). No hubo significancia para la asociación de E. faecalis con condición alguna.

\section{Tratamiento y evolución}

La terapia antimicrobiana incluyó múltiples alternativas en el caso de los pacientes con ED piógena, dentro de ellas, cefalosporinas, quinolonas, glicopéptidos y sulfonamidas, generalmente asociados, los que en promedio se indicaron por 63,8 días (IQR 53-72 días).

Los tres casos de ED tuberculosa se trataron con esquema asociado de tres fármacos anti-tuberculosos (isoniazida, rifampicina, pirazinamida), según la norma nacional vigente en ese momento ${ }^{10}$.

Siete pacientes $(18,9 \%)$ presentaron reacciones adversas asociadas (RAM) al uso de antimicrobianos, incluyendo alergias cutáneas y alteraciones del perfil hepático. De hecho, dos de los tres pacientes con Mal de Pott presentaron RAM hepática. Ninguna RAM fue grave ni con resultado fatal.

Cerca de $60 \%$ recibió exclusivamente terapia antimicrobiana y $40 \%$ requirió asociarle tratamiento quirúrgico, incluyendo aseo quirúrgico, descompresión, injerto óseo y/o fijación de columna; en cuatro pacientes se requirió dos o más intervenciones quirúrgicas por ED (Tabla 5).

Catorce pacientes $(37,8 \%)$ presentaron complicaciones durante su estadía hospitalaria, no directamente relacionadas con la ED, incluyendo 10 de origen infeccioso: dos casos de infecciones del torrente sanguíneo asociada a CVC (por S. epidermidis MR y Stenotrophomonas maltophilia, respectivamente), dos infecciones urinarias asociadas a catéter urinario permanente por E. coli, dos infecciones superficiales de sitio quirúrgico (en pacientes operados de columna por ED), una infección por Clostri-

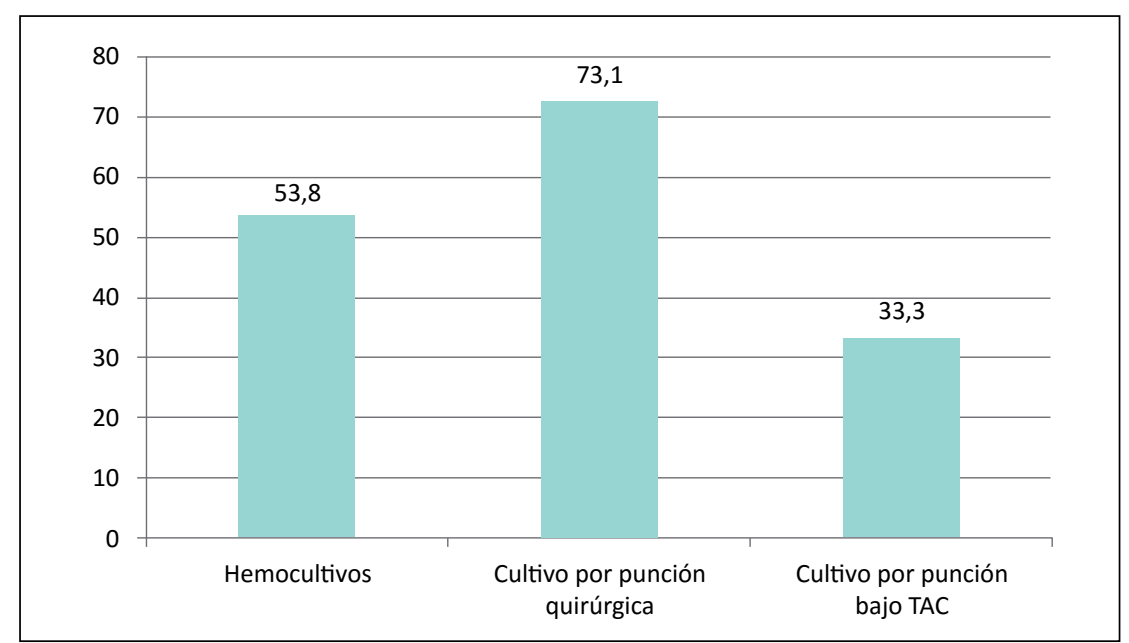

Figura 1. Rendimiento (\%) de los distintos métodos microbiológicos en 25 pacientes con espondilodiscitis piógena confirmada microbiológicamente, en el HMS. TAC: tomografía axial computada.

Tabla 5. Aspectos terapéuticos y evolutivos en 37 pacientes con espondilodiscitis, atendidos en el Hospital Militar de Santiago entre 2006 y 2013

\begin{tabular}{lcc}
\hline Tratamiento y evolución & $\mathbf{n} / \mathbf{N}$ & $\%$ \\
Aspectos terapéuticos & & \\
Sólo antimicrobianos & $22 / 37$ & 59,5 \\
Antimicrobianos + cirugía & $15 / 37$ & 40,5 \\
Un acto quirúrgico & $11 / 37$ & 29,7 \\
Dos actos quirúrgicos & $3 / 37$ & 8,1 \\
Tres o más actos quirúrgicos & $1 / 37$ & 2,7 \\
Complicaciones y evolución & & \\
Complicaciones intrahospitalarias & $14 / 37$ & 37,8 \\
Efectos adversos a antimicrobianos & $7 / 37$ & 18,9 \\
Secuelas neurológicas & $7 / 37$ & 18,9 \\
Rehospitalización & $9 / 37$ & 24,3 \\
Fallecidos & 0 & 0 \\
\hline
\end{tabular}

dium difficile, dos diarreas asociadas a antibioterapia y una queratitis herpética. Además se observaron cinco pacientes con complicaciones no infecciosas: uno con trastorno adaptativo asociado a hospitalización prolongada, un episodio de hemorragia digestiva por úlcera duodenal, un íleo médico, una hiperglicemia de difícil control y un paciente con descompensación de insuficiencia cardíaca.

Un 18,9\% quedó con secuelas neurológicas, principalmente compromiso motor de las extremidades inferiores, manifestado por paresia o paraplejia (manifestaciones todas confirmadas por neurólogo y/o neurocirujano). Esta discapacidad motora no se asoció significativamente a la presencia de fiebre, baja de peso, co-morbilidad de algún 
tipo, cirugía de columna, adquisición nosocomial versus comunitaria ni al agente etiológico.

Por otra parte, nueve pacientes ameritaron más de una hospitalización por necesidad de un nuevo estudio, tratamiento o complicaciones asociadas. No hubo fallecidos durante la estadía hospitalaria (Tabla 5).

\section{Discusión}

La ED u osteomielitis vertebral es una enfermedad infrecuente, pero en aumento en las últimas décadas, cuya incidencia se ha estimado entre 2,2 y 5,8 por cada 100.000 hbts/año ${ }^{11-13}$. Posee elevada morbilidad, por lo que demanda una alta sospecha y diagnóstico precoz integrando elementos clínicos, de laboratorio e imagenológicos.

En esta serie se demostró que los pacientes mayormente afectados fueron hombres y de edad avanzada, lo que resulta concordante con series internacionales ${ }^{13-15}$ y nacionales ${ }^{9}$, pero evidenciando un cambio respecto a reportes más antiguos, donde el grupo más afectado eran mujeres adultas jóvenes ${ }^{7}$, probablemente en contexto de cambios demográficos de países en desarrollo, como Chile. Se comprobó un período de evolución prolongado y un alto porcentaje ( $>90 \%$ ) de alguna co-morbilidad o condición de riesgo para ED, siendo diabetes mellitus la más frecuente de ellas, lo que ya ha sido observado previamente $e^{14,16,17}$, y que en parte puede explicar la mayor tasa de agentes bacterianos piogénicos como causales, asociados en general a dichas condición. No se objetivó la presencia de pacientes con neutropenia, infección por VIH ni neoplasias hematológicas.

Se constató además períodos de larga estadía hospitalaria, en relación a una tardía sospecha clínica y demoras en la realización de estudios y a lo prolongado de las terapias antimicrobianas y/o quirúrgicas, hallazgos reconocidos en múltiples ocasiones $5,7,8,11,12$.

La ED se caracteriza por la presencia de un dolor axial, asociado o no a fiebre, síntomas constitucionales y/o neurológicos ${ }^{1,2,16}$. Nuestra serie confirma que las manifestaciones clínicas más relevantes fueron el dolor axial, la fiebre y los síntomas neurológicos, hallazgos constantemente presente en múltiples series ${ }^{5,7,11,12}$. Del total de las ED, se describe hasta $25 \%$ de adquisición nosocomial o asociado a atención en salud, la mayoría por inoculación directa posterior a una cirugía de columna, en pacientes con más co-morbilidades, y con mayor riesgo de complicaciones, recaídas y mortalidad ${ }^{2,18}$.

La PCR y la VHS estuvieron elevadas en la mayorías de los pacientes, mientras que sólo aproximadamente un tercio presentó leucocitosis. Estos hallazgos son concordantes con otros reportes ${ }^{7,9,14}$. La PCR ha sido reconocida como un importante marcador de respuesta a tratamiento. En contraste, la utilidad de la VHS para el seguimiento es controvertido. En un estudio pequeño, 50\% (9/18) de los pacientes con VHS estacionaria presentaron adecuada respuesta a tratamiento ${ }^{19}$, mientras que otro estudio en 45 pacientes reveló que valores superiores a $50 \mathrm{~mm} / \mathrm{h}$ están asociado a mayor riesgo de fracaso terapeútico ${ }^{20}$. Por ello, las alteraciones en los valores plasmáticos de estos marcadores inflamatorios deben ser interpretadas con cautela.

La radiología convencional, pese a estar ampliamente disponible, tiene una muy baja sensibilidad para el diagnóstico de ED y muestra sólo alteraciones, al menos tres a cuatro semanas tras el inicio de los síntomas. En contraste, la cintigrafía ósea con radioisótopo (Tc-99m) es ampliamente utilizada por su relativa disponibilidad y escaso daño colateral, dado que prescinde de medios contrastados o gadolinio. Posee una sensibilidad elevada, superior a $70 \%$ y detecta alteraciones de manera independiente del tiempo de evolución de la enfermedad. Sin embargo, su valor predictor negativo es bajo y su especificidad no supera a $60 \%{ }^{21}$. En una serie nacional de 18 casos, el estudio cintigráfico mostró actividad osteoblástica en $100 \%$ de los casos 7 . Estos resultados son concordantes con la alta sensibilidad observada en esta serie (94\%), lo que convierte a la cintigrafía en un método imagenológico útil ante la sospecha de ED, incluyendo aquellas que llevan pocos días de evolución clínica ${ }^{7-21}$.

La TAC ofrece una menor sensibilidad respecto a los métodos cintigráficos, pero permite evaluar con mayor precisión la existencia de colecciones, el grado de destrucción vertebral, otras complicaciones, y facilita la evaluación de la estabilidad de la columna. Además, permite realizar punciones percutáneas. Su sensibilidad se ha reportado cercana a $80 \% 0^{7,16,22}$, y su especificidad puede alcanzar hasta $90 \%{ }^{7,16,22}$. En nuestra serie su rendimiento fue menor.

Por otro lado, la RM tiene una especificidad y sensibilidad cercanas a $100 \%$ y es considerada la técnica imagenológica de elección ante la sospecha de ED, por su capacidad en confirmar el diagnóstico, sus complicaciones y su localización ${ }^{23-25}$. Su disponibilidad en Chile se ha incrementado en las últimas décadas, aunque existen aún limitaciones para su acceso. En nuestro trabajo la RM siempre confirmó el diagnóstico de ED (100\% sensibilidad).

Finalmente, en los últimos años ha ido aumentado la experiencia con tomografía de emisión de positrones (PET) con F18-FDG. Posee un rendimiento comparable con la RM (sensibilidad 97\%, especificidad 88\%), lo que la convierte en una excelente alternativa para pacientes con implantes metálicos u otras contraindicaciones a la $\mathrm{RM}^{4}$. Su aplicación conjunta con la tomografía computada (PET-scan) aumenta su rendimiento ${ }^{4}$. No obstante, tiene un alto costo y no se encuentra ampliamente disponible en nuestro medio ${ }^{2,26,27}$. En esta serie, en ningún paciente se realizó una PET. 
Diferentes agentes etiológicos han sido descritos en la literatura científica como causa de ED. Su frecuencia relativa está influenciada por las características de la población estudiada, la que puede variar en edad, antecedentes quirúrgicos, co-morbilidades crónicas o inmunocompromiso. Las causas más frecuentes descritas corresponden a Staphylococcus spp., Streptococcus spp., M. tuberculosis, enterobacterias y algunos bacilos gramnegativos no fermentadores. En nuestra serie, el principal agente infeccioso aislado fue $S$. aureus, lo que es concordante con la mayoría de las series y reportes internacionales, en posible asociación con algunos factores de riesgo para infecciones estafilocóccicas invasoras, como diabetes mellitus, la presencia de dispositivos invasores y la cirugía reciente, con o sin prótesis ${ }^{5,14,15,16,28}$; en la actualidad se describe como el agente infeccioso más importante de ED, con frecuencias entre 20 y $84 \%{ }^{1}$. La asociación de ED con E. faecalis está descrita en la literatura científica en forma menos relevante, con frecuencias estimadas entre $5-20 \%$ del total de ED. No obstante, en nuestra serie fue segundo en frecuencia luego de $S$. aureus, describiéndose en la mayoría de los reportes que la vía urinaria es la puerta de entrada hacia el torrente sanguíneo y por consiguiente a la vértebra, con o sin endocarditis ${ }^{1}$. En nuestra serie, la presencia de E. faecalis no tuvo factores de riesgo significativos para su presentación como agente causal.

La ED tuberculosa fue reconocida en este reporte como una etiología poco frecuente, sin mayores diferencias clínicas ni de laboratorio con respecto al resto a los pacientes con ED piógenas, aunque el reducido número de los pacientes con Mal de Pott en esta serie no permite hacer mayores análisis. La baja incidencia de TBC vertebral revela un marcado contraste respecto a series chilenas anteriores ${ }^{6,7}$ y también a algunas europeas ${ }^{28-31}$, en relación a la declinación progresiva de la incidencia de TBC en países en vías de desarrollo y desarrollados, como Chile. Por lo tanto es esperable que aún aparezcan esporádicos nuevos casos de ED tuberculosa, que fue señalada hace años como la forma más frecuente de la TBC osteoarticular en nuestro país ${ }^{32}$. Se pesquisó sólo un caso con cultivo de Koch positivo para M. tuberculosis, lo que obliga a recurrir al estudio histológico o molecular para el diagnóstico. La RPC convencional ha sido utilizada exitosamente en algunos estudios para el diagnóstico de ED tuberculosa, con una sensibilidad reportada hasta $94 \%$ y una especificidad de $83 \%^{5,33}$; no obstante, la mayoría de los estudios que evalúan los métodos moleculares en pacientes con TBC extra-pulmonar incluyen pocos pacientes con Mal de Pott ${ }^{34,35}$. No existen a la fecha estudios que evalúen la utilidad de técnicas moleculares en tiempo real automatizadas como Xpert MTB/RIF®, de gran utilidad y ampliamente recomendadas para el diagnóstico de la TBC extra-pulmonar ${ }^{36,37}$, y que en un futuro podrían mejorar el rendimiento del diagnóstico molecular en ED. En nuestro caso sólo fue realizada RPC convencional para M. tuberculosis en un caso, la que fue negativa.

La diversidad de etiologías en este trabajo y en otros, impide plantear esquemas empíricos de tratamiento antimicrobiano y obliga a realizar un estudio dirigido. El acercamiento inicial para la obtención del agente etiológico es a través de la toma de hemocultivos ${ }^{4}$, idealmente aerobios y anaerobios estrictos; sin embargo, su sensibilidad es variable, y no va más allá de $40-60 \%{ }^{1,2}$. El método de elección, es el estudio de la lesión vertebral, que idealmente debe incluir un análisis microbiológico e histológico. Dependiendo de las circunstancias locales, éste puede ser obtenido por técnicas percutáneas bajo radiología o por cirugía mínima abierta. En nuestra serie el mayor rendimiento diagnóstico lo obtuvo la punción abierta de la vértebra y/o disco, por sobre los hemocultivos de sangre periférica o la punción percutánea bajo scanner, lo que es comparable a lo reportado en la literatura científica, aunque comparativamente con una mayor sensibilidad de los hemocultivos ${ }^{1,2,11,38}$.

El manejo de estos pacientes contempla la inmovilización, a través del uso de corsé y reposo, la administración de antimicrobianos y la cirugía en casos seleccionados. La mayoría de las recomendaciones proviene de estudios retrospectivos y series con tamaño de muestra limitado por la baja frecuente de esta enfermedad.

Para los casos de ED se recomienda el uso de esquemas antimicrobianos de tipo bactericida, en forma prolongada y con adecuada penetración ósea para asegurar la erradicación microbiológica y evitar recaídas. La aparición de antimicrobianos con presentación oral con alta biodisponibilidad y concentraciones tisulares adecuadas como son fluoroquinolonas y sulfas, ha permitido el tratamiento de esta condición con esquemas orales, en altas dosis. Por ejemplo, algunas fluoroquinolonas, presentan biodisponibilidad alrededor de $90 \%{ }^{39}$, pueden alcanzar concentraciones óseas varias veces superiores a los observados en el plasma ${ }^{39,40}$ y poseen excelente actividad intracelular, limitando con ello las recaídas en infecciones óseas y articulares ${ }^{40}$. En el caso de moxifloxacina, se administra una vez al día y su metabolismo predominantemente hepático permite su uso en pacientes con función renal deteriorada. Sin embargo, el uso de ésta y otras fluoroquinolonas tiene un riesgo consecuente de diarrea asociada a antimicrobianos, incluyendo infección por $C$. difficile, riesgo de interacciones medicamentosas y daño colateral, entre otros ${ }^{4,39}$. Gracias a su alta biodisponibilidad y altas concentraciones en hueso, clindamicina ha sido considerada para tratar infecciones por $S$. aureus por vía oral; sin embargo, su acción sobre $S$. aureus es bacteriostática y su uso se asocia a alta incidencia de diarrea. La penicilinas anti-estafilocócicas orales poseen utilidad limitada por su baja biodisponibilidad, baja penetración 
ósea y significativo riesgo de reacciones adversas con uso prolongado $^{41,42}$. Cotrimoxazol es una alternativa teórica para el tratamiento de la osteomielitis crónica, incluyendo ED asociadas a $S$. aureus o coagulasa negativa, resistentes o no a oxacilina, y a enterobacterias susceptibles, pero hay sólo reportes anecdóticos que han ensayado esta alternativa; es una molécula de alta biodisponibildad oral, penetración y llegada al tejido óseo, de presentación oral y bajo costo ${ }^{43}$.

La terapia oral es la forma establecida de tratamiento en pacientes con Mal de Pott, actualmente con esquemas asociados de cuatro fármacos y prolongados ${ }^{44}$. En este caso, los tres pacientes fueron tratados según la norma nacional previamente vigente ${ }^{10}$, que sugería la asociación de tres fármacos anti-tuberculosos, sin etambutol, para casos de TBC extra-pulmonares o pulmonares no bacilíferas.

No se conoce la duración mínima del tratamiento antimicrobiano ni la vía de administración óptima en las ED piógenas. En nuestro estudio fueron tratados durante nueve semanas en promedio, concordante con la literatura médica en que se recomienda una duración mínima de 6-8 semanas ${ }^{45,46}$. Sin embargo, en un estudio observacional en que se trató exclusivamente por vía intravenosa a 108 pacientes durante cinco semanas (promedio) no se evidenció una mayor mortalidad, respecto a series con tratamientos más prolongados ${ }^{27}$. Un estudio reciente, randomizado y controlado, revela la no inferioridad del tratamiento antimicrobiano de la ED piógena por 6 versus 12 semanas $^{47}$.

En cuanto al mejor momento de traspaso a vía oral, recientemente, un estudio retrospectivo y de pequeño tamaño de muestra no observó recaídas ni resultados adversos luego de tratar durante dos semanas de forma intravenosa seguida por terapia oral y la PCR fue un predictor de manera independiente del mejor momento de traspaso a terapia oral ${ }^{48}$.

El manejo quirúrgico está indicado en caso de un absceso mayor, compresión medular o de raíz con compromiso neurológico significativo, destrucción vertebral que evolucione con deformidad raquídea, fracaso en el tratamiento médico o dolor intratable ${ }^{4,24,49}$. El pronóstico de los pacientes sometidos a cirugía es al menos similar a aquellos seleccionados para manejo médico ${ }^{3,17,50}$. De manera similar a otras series ${ }^{7,14,27}$, un alto porcentaje de nuestros pacientes $(40 \%)$ requirió algún tipo de intervención quirúrgica incluyendo aseo quirúrgico, fijación y descompresión. Aún no está claro cuál es la mejor estrategia en pacientes con síntomas neurológicos con indicación quirúrgica, y que al mismo tiempo poseen edad avanzada o con co-morbilidad significativa. Si bien la terapia conservadora mostró en un estudio retrospectivo remisión de los síntomas neurológicos en 73\% de pacientes de edad avanzada ${ }^{17}$, para otros autores la edad no debiese contraindicar la cirugía e incluso podría mejorar la calidad de vida ${ }^{51}$. Es por ello, que la decisión de una intervención quirúrgica debe ser individualizada y multidisciplinaria.

No hubo letalidad asociada en nuestra serie, a diferencia de reportes previos que revelan porcentajes bajos de fallecidos (5-8\%) por bacteriemia, sepsis o complicaciones asociadas a estadías hospitalarias prologadas ${ }^{7,16,17}$. No obstante, recientemente se ha observado un aumento en la mortalidad a largo plazo, especialmente durante el primer año ${ }^{52,53}$. Al igual que en otras series ${ }^{14-16,54}$, hubo un porcentaje considerable de complicaciones o secuelas neurológicas y funcionales, las que no tuvieron relación a la localización. Sin embargo, el único paciente con ED de columna cervical, evolucionó con secuela de tipo motora. Interesantemente, esta localización ha sido asociada a peor pronóstico 3,9 .

\section{Conclusiones}

La mayoría de los pacientes con ED de esta serie correspondieron a adultos mayores, con hospitalizaciones prolongadas y condiciones de riesgo asociadas, con una elevada etiología bacteriana piógena, principalmente $S$. aureus y una baja frecuencia de $\mathrm{TBC}$, lo que representa un cambio epidemiológico relevante. Llamó la atención la aparición de cepas de E. faecalis. Se logró precisar el agente etiológico con una frecuencia mayor a lo revelado en otras publicaciones, con el estudio dirigido vía abierta como el de mejor rendimiento. El uso de la RM de columna fue fundamental como examen confirmatorio y localizador. Aunque no hubo letalidad asociada, existió un considerable número de secuelas neurológicas. La ED sigue siendo una entidad infrecuente y un desafío diagnóstico multidisciplinario, que aún implica elevada morbilidad y prolongadas estadías hospitalarias.

\section{Resumen}

Introducción: La espondilodiscitis (ED) implica prolongados períodos de hospitalización, de latencia diagnóstica y riesgo de complicaciones a largo plazo. No existen publicaciones recientes en Chile al respecto. Objetivos: Caracterizar un grupo de pacientes con ED. Pacientes y Métodos: Serie clínica, que incluyó pacientes en un período de ocho años. Resultados: 37 pacientes, $37,8 \%$ mujeres y $62,2 \%$ hombres, con promedio etario 66,8 años; $64,9 \%$ adultos mayores, $35,1 \%$ diabéticos y $21,6 \%$ con co-morbilidad urológica. Los principales síntomas fueron dolor y fiebre. 89,2\% tuvo elevación de VHS. $86,5 \%$ contó con resonancia magnética, que siempre fue confirmatoria, siendo la columna lumbar la localización más frecuente (43,2\%). Se identificó etiología en $28 / 37$ pacientes: en $71,4 \%$ cocáceas grampositivas 
(Staphylococcus aureus predominantemente), sólo en 10,7\% M. tuberculosis. Staphylococcus aureus estuvo asociado a co-morbilidades médicas en forma significativa $(\mathrm{p}<0,05)$ y el grupo de bacilos gramnegativos a historia hepatobiliar y/o intestinal $(\mathrm{p}<0,05)$. El método de mayor rendimiento fue el cultivo obtenido por punción quirúrgica. El tratamiento antimicrobiano fue indicado en promedio por 63,8 días (IQR 53-72), con reacciones adversas en 18,9\%. La estadía hospitalaria fue 38,9 días promedio, no existiendo fallecidos durante este período. $18,9 \%$ presentó secuelas motoras. Discusión: La mayoría de pacientes con ED correspondió a adultos mayores, siendo $S$. aureus la principal etiología. Hubo una baja frecuencia de $M$. tuberculosis. Resultó considerable la magnitud de efectos adversos asociados a la terapia antimicrobiana y las complicaciones neurológicas.

\section{Referencias bibliográficas}

1.- Gouliouris T, Aliyu S H, Brown N M. Spondylodiscitis: update on diagnosis and management. J Antimicrob Chemother 2010; 65 Suppl 3: iii11-24.

2.- Zimmerli W. Vertebral osteomyelitis. N Engl J Med 2010; 362: 1022-9.

3.- Hadjipavlou A G, Mader J T, Necessary J T, Muffoletto A J. Hematogenous pyogenic spinal infections and their surgical management. Spine (Phila, Pa 1976) 2000; 25: 1668-79.

4.- Berbari E F, Kanj S S, Kowalski T J, Darouiche R O, Widmer A F, Schmitt S K, et al Infectious Diseases Society of America (IDSA) clinical practice guidelines for the diagnosis and treatment of native vertebral osteomyelitis in adults. Clin Infect Dis 2015; 61 (6): e26-46.

5.- Aagaard T, Roed C, Dragsted C, Skinhøj P. Microbiological and therapeutic challenges in infectious spondylodiscitis: a cohort study of 100 cases, 2006-2011. Scand J Infect Dis 2013; 45 (6): 417-24.

6.- Cottle L, Riordan T. Infectious spondylodiscitis. J Infect 2008; 56 (6): 401-12.

7.- Fica A, Bozán F, Aristegui M, Bustos P. Espondilodiscitis. Análisis de una serie de 25 casos Rev Med Chile 2003; 131: 473-82.

8.- Eugenin O. Espondilodiscitis piógena: experiencia prospectiva. Rev Chil Ortop Traumatol 1998; 39: 15-26.

9.- Urrutia J, Zamora T, Campos M. Cervical pyogenic spinal infections: are they more severe diseases than infections in other vertebral locations? Eur Spine J 2013; 22 (12): 2815-20.

10.- Programa Nacional de Control de la Tuberculosis. Manual de organización y normas técnicas. Ministerio de Salud, Gobierno de Chile, 2005.

11.- Krogsgaard M R, Wagn P, Bengtsson J. Epidemiology of acute vertebral osteomyelitis in Denmark: 137 cases in Denmark 1978-1982, compared to cases reported to the National Patient Register 1991-1993. Acta Orthop Scand 1998; 69: 513-7.

12.- Beronius M, Bergman B, Andersson R. Vertebral osteomyelitis in Goteborg, Sweden: a retrospective study of patients during 19901995. Scand J Infect Dis 2001; 33: 527-32.
13.- Kehrer M, Pedersen C, Jensen T G, Lassen A T. Increasing incidence of pyogenic spondylodiscitis: A 14-year population-based study. J Infect 2014; 68 (4): 313-20.

14.- Kaya S, Ercan S, Kaya S, Aktas U, Kamasak K, Ozalp H, et al. Spondylodiscitis: evaluation of patients in a tertiary hospital. J Infect Dev Ctries 2014; 8 (10): 1272-6.

15.- D'Agostino C, Scorzolini L, Massetti A P, Carnevalini M, d'Ettorre G, Venditti M, et al. A seven-year prospective study on spondylodiscitis: epidemiological and microbiological features. Infection 2010; 38 (2): 102-7.

16.- Mylona E, Samarkos M, Kakalou E, Fanourgiakis P, Skoutelis A. Pyogenic vertebral osteomyelitis: a systematic review of clinical characteristics. Semin Arthritis Rheum 2009; 39: 10-7.

17.- Yoshimoto M, Takebayashi T, Kawaguchi S, Tsuda H, Ida K, Wada T, et al. Pyogenic spondylitis in the elderly: a report from Japan with the most aging society. Eur Spine J 2011; 20 (4): 649-54.

18.- Pigrau C, Rodríguez-Pardo D, FernándezHidalgo N, Moretó L, Pellise F, Larrosa M, et al. Healthcare associated hematogenous pyogenic vertebral osteomyelitis. Medicine 2015; 94 (3): e365.

19.- Carragee E J, Kim D, van der Vlugt T, Vittum D. The clinical use of erythrocyte sedimentation rate in pyogenic vertebral osteomyelitis. Spine (PhilaPa 1976) 1997; 22 (18): 2089-93.

20.- Yoon S H, Chung S K, Kim K J, Kim H J, Jin Y J, Kim H B. Pyogenic vertebral osteomyelitis: identification of microorganism and laboratory markers used to predict clinical outcome. Eur Spine J 2010; 19 (4): 575-82.

21.- Lisbona R, Derbekyan J, Novales-Díaz J, Veksler A. Gallium-67 scintigraphy in tuberculosis and nontuberculous infectious spondylitis. J Nucl Med 1993; 34: 853-9.

22.- Rivero M G, Salvatore A J, Wouters L. Espondilodiscitis infecciosa espontánea en adultos. Análisis de 30 casos. Medicina (Bs Aires) 1999; 59: 143-50.

23.- Leone A, Dell'Atti C, Magarelli N, Colelli P, Balanika A, Casale R, et al. Imaging of spondylodiscitis. Eur Rev Med Pharmacol Sci
2012; 16 (Suppl 2): 8-19.

24.- Diehn F E. Imaging of spine infection. Radiol Clin North Am 2012; 50 (4): 777-98.

25.- Duarte R, Vaccaro A. Spinal infection: state of the art and management algorithm. Eur Spine J 2013; 22: 2787-99.

26.- Prodromou M L, Ziakas P D, Poulou L S, Karsaliakos P, Thanos L, Mylonakis E. FDG PET is a robust tool for the diagnosis of spondylodiscitis: a meta-analysis of diagnostic data. Clin Nucl Med 2014; 39 (4): 330-5.

27.- Palestro C J. Radionuclide imaging of osteomyelitis. Semin Nucl Med 2015; 45 (1): 32-46.

28.- Cebrián Parra J L, Saez-Arenillas Martín A, Urda Martínez-Aedo A L, Soler Ivañez I, Agreda E, López-Durán Stern L. Management of infectious discitis. Outcome in one hundred and eight patients in a university hospital. Int Orthop 2012; 36 (2): 239-44.

29.- Joughin E, McDougall C, Parfitt C, Yong-Hing K, Kirkaldy-Willis W H. Causes and clinical management of vertebral osteomyelitis in Saskatchewan. Spine 1991; 16 (3): 261-4.

30.- Belzunegui J, Del Val N, Intxausti J J, De Dios J R, Queiro R, González C, et al. Vertebral osteomyelitis in northern Spain. Report of 62 cases. Clin Exp Rheumatol 1999; 17 (4): 447-52.

31.- Grammatico L, Baron S, Rusch E, Lepage B, Surer N, Desenclos J C, et al. Epidemiology of vertebral osteomyelitis (VO) in France: analysis of hospital-discharge data 2002-2003. Epidemiol Infect 2008; 136 (5): 653-60.

32.- Valdivia P, Álvarez F, Storme O, Hidalgo C, Pinochet C, Mellado M, et al. Tuberculosis osteoarticular Región Sur Chile 1981-1990. Rev Chil Ortop Traumatol 1992; 33: 58-64.

33.- Berk R H, Yazici M, Atabey N, Ozdamar O S, Pabuccuoglu U, Alici E. Detection of Mycobacterium tuberculosis in formaldehyde solution-fixed, paraffin-embedded tissue by polymerase chain reaction in Pott's disease. Spine 1996; 21 (17): 1991-5.

34.- Causse M, Ruiz P, Gutiérrez-Aroca J B, Casal M. Comparison of two molecular methods for rapid diagnosis of extrapulmonary tuberculosis. J Clin Microbiol 2011; 49: 3065-7. 
35.- Cheng V C, Yam W C, Hung I F, Woo P C, Lau S K, Tang B S, et al. Clinical evaluation of the polymerase chain reaction for the rapid diagnosis of tuberculosis. J Clin Pathol 2004; 57: 281-5.

36.- Lawn S D, Zumla A L. Diagnosis of extrapulmonary tuberculosis using the Xpert ${ }^{\circledR}$ MTB/RIF assay. Expert Rev Anti Infect Ther 2012; 10 (6): 631-5.

37.- Denkinger C M, Schumacher S G, Boehme C C, Dendukuri N, Pai M, Steingart K R. Xpert MTB/ RIF assay for the diagnosis of extrapulmonary tuberculosis: a systematic review and meta-analysis. Eur Respir J 2014; 44 (2): 435-46.

38.- Hegde V, Meredith D S, Kepler C K, Huang R C. Management of postoperative spinal infections. World J Orthop 2012; 3 (11): 182-9.

39.- Rodvold K A, Neuhauser M. Pharmacokinetics and pharmacodynamics of fluoroquinolones. Pharmacotherapy 2001; 21: S233-52.

40.- Bergan T. Pharmacokinetics of the fluorquinolones. En: Andriole V (ed). The Quinolones (second edition), Academic Press, California 1998; 144-82.

41.- Valour F, Trouillet-Assant S, Riffard N, Tasse J, Flammier S, Rasigade J P, et al. Antimicrobial activity against intraosteoblastic Staphylococcus aureus. Antimicrob Agents Chemother 2015; 59 (4): 2029-36.

42.- Valour F, Karsenty J, Bouaziz A, Ader F,
Tod M, Lustig S, et al. Antimicrobial-related severe adverse events during treatment of bone and joint infection due to methicillinsusceptible Staphylococcus aureus. Antimicrob Agents Chemother 2014; 58 (2): 746-55.

43.- Grim S A, Rapp R P, Martin C A, Evans M E. Trimethoprim-sulfamethoxazole as a viable treatment option for infections caused by methicillin-resistant Staphylococcus aureus. Pharmacotherapy 2005; 25: 253-64.

44.- Normas técnicas para el control y eliminación de la tuberculosis. Ministerio de Salud, Gobierno de Chile, 2014.

45.- Legrand E, Flipo R M, Guggenbuhl P, Masson C, Maillefert J F, Soubrier M. Management of nontuberculous infectious discitis. Treatments used in 110 patients admitted to 12 teaching hospitals in France. Joint Bone Spine 2001; 68: 504-9.

46.- Cheung W Y, Luk K D. Pyogenic spondylodiscitis. Int Orthop 2012; 36 (2): 397-404.

47.- Bernard L, Dinh A, Ghout I, Simo D, Zeller V, Issartel B, et al. Antibiotic treatment for 6 weeks versus 12 weeks in patients with pyogenic vertebral osteomyelitis: an open-label, non-inferiority, randomised, controlled trial. Lancet 2015; 385 (9971): 875-82.

48.- Babouee Flury B, Elzi L, Kolbe M, Frei R, Weisser M, et al. Is switching to an oral antibiotic regimen safe after 2 weeks of intravenous treatment for primary bacterial vertebral osteomyelitis? BMC Infect Dis 2014; 14: 226 .

49.- Chen W H, Jiang L S, Dai L Y. Surgical treatment of pyogenic vertebral osteomyelitis with spinal instrumentation. Eur Spine J 2007; 16 (9): 1307-16.

50.- Malawski S K, Lukawski S. Pyogenic infection of the spine. Clin Orthop 1991; 272: 58-66.

51.- Sobottke R, Röllinghoff M, Zarghooni K, Zarghooni K, Schlüter-Brust K, Delank K S, et al. Spondylodiscitis in the elderly patient: clinical mid-term results and quality of life. Arch Orthop Trauma Surg 2010; 130 (9): 1083-91.

52.- Aagaard T, Roed C, Larsen A R, Petersen A, Dahl B, Skinhøj P, et al. Long-term mortality after Staphylococcus aureus spondylodiscitis: a Danish nationwide population-based cohort study. J Infect 2014; 69 (3): 252-8.

53.- Kehrer M, Pedersen C, Jensen T G, Hallas J, Lassen A T. Increased short-and long-term mortality among patients with infectious spondylodiscitis compared with a reference population. Spine J 2015; 15 (6): 1233-40.

54.- Urrutia J, Campos M, Zamora T, Canessa V, García P, Briceño J. Does pathogen identification influence clinical outcomes in patients with pyogenic spinal infections? J Spinal Disord Tech 2015; 7: E417-21. 